\title{
La Educación y la Teoría del Reconocimiento: entrevista a Axel Honneth
}

Francesc J. Hernàndez I Dobon'

Benno Herzog'

Maurício Rebelo Martins"

'Universidad de Valencia, Valencia - España

"Universidade Estadual de Campinas (UNICAMP), Campinas/SP - Brasil

Cuando hoy nos preguntamos por la actualidad de la Teoría Crítica, por la presencia de los pensadores de la Escuela de Frankfurt, encontramos un referente en la obra del Axel Honneth. Filósofo y sociólogo alemán, director desde 2001 del Instituto de Investigaciones Sociales de la Universidad de Frankfurt, profesor de la Universidad de Columbia en los Estados Unidos, es considerado hoy uno de los representantes más importantes de la Escuela de Frankfurt. La Teoría del Reconocimiento de Axel Honneth tiene cada vez mayor importancia en los círculos académicos anglosajones, europeos y latinoamericanos.

Honneth, al igual que Jürgen Habermas, presenta su propia posición teórica en comparación con sus predecesores. En este sentido, en primer lugar, se trata de mostrar los límites de la Teoría de la Acción Comunicativa de Habermas para, a continuación, formular su propia posición teórica que se puede resumir como Teoría del Reconocimiento. Se utiliza el reconocimiento como la herramienta conceptual más adecuada para desentrañar las experiencias sociales de injusticia y para comprender la fuente motivacional de las luchas sociales.

El tema central de esta conversación elige explorar el lugar que la educación ocupa en su Teoría del Reconocimiento. Entre los filósofos de su generación es, posiblemente, uno de los que ha ofrecido mayor cantidad de entrevistas a interlocutores de los más variados terrenos. Sin embargo, las cuestiones relativas a la educación y la formación no

Educação \& Realidade, Porto Alegre, v. 42, n. 1, p. 395-406, ene./mar. 2017. 395 http://dx.doi.org/10.1590/2175-623670297 
habían tenido un papel destacado en la obra de Honneth hasta poco tiempo. Esta es una oportunidad para conocer no sólo un poco acerca de lo que piensa Honneth de la educación, sino también de su experiencia como estudiante y profesor. La entrevista fue realizada por medios electrónicos durante el año 2015 de la Universidad de Valencia en España. Cabe señalar su atención y generosidad para responder a las preguntas de propia mano.

Francesc Hernàndez (F. H.), Benno Herzog (B. H.) \& Maurício Rebelo (M. R.): Estimado profesor Honneth, si echa la vista atrás puede contemplar una rica experiencia docente en diferentes universidades y sistemas educativos. Al contrario de su papel como filósofo e investigador social, su trabajo docente es poco conocido por el público. ¿Qué importancia tenía y tiene la teoría del reconocimiento, tal como usted la ha desarrollado a lo largo de su vida, para su práctica como docente?

Axel Honneth: La historia de mi elaboración de la teoría del reconocimiento en la educación y la formación comienza mucho antes, a saber, en mi experiencia como estudiante en el instituto y en la universidad. A diferencia del sistema de enseñanza norteamericano - en la medida en que puedo enjuiciarlo-, el sistema alemán de enseñanza media dirigida a la universidad opera al principio de manera muy intensa con mensajes desalentadores, al señalar de manera informal a los alumnos y a las alumnas que apenas disponen de talentos y capacidades propias, y que tendrían que aprender todo ello en una enseñanza futura. Al contrario de lo que sucede en el sistema alemán, en el norteamericano el mensaje es al principio afirmativo. En este, el mensaje oculto es: "Tú" eres capaz, tienes muy buenas aptitudes y tienes que desarrollarlas mediante la enseñanza. Que el sistema alemán opere con aquel principio no sería la única razón que me convirtió en un alumno nefasto en el instituto, pero aportó su grano de arena porque nunca pude desarrollar la impresión de que llevara conmigo aptitudes valiosas. En la universidad, la situación fue similar al principio: estaba completamente callado como un ratoncillo. Estaba sentado en las clases universitarias y no lograba abrir la boca porque nadie me inspiraba confianza. Toda mi energía se desplazó a la redacción de trabajos escritos que, por regla general, resultaron muy buenos. Esta situación no cambió hasta que me encontré con un catedrático que disponía de una gran capacidad para dar confianza a sus estudiantes en el desarrollo de sus propias ideas y representaciones teóricas. El hombre del que hablo era el sociólogo Urs Jaeggi, en aquel entonces un docente universitario altamente capacitado, no porque se hubiera dedicado a la didáctica, sino porque disponía de un talento adquirido para despertar capacidades y talentos ocultos. También Habermas, al que conocí más tarde cuando fui su asistente, podía tener tales rasgos cuando había adquirido confianza en las capacidades de sus estudiantes.

Cuento todo esto para dejar claro que el papel del reconocimiento en la enseñanza y en las clases universitarias brotó en mí ya de una forma preteórica, por así decirlo, y desde luego solo de una forma implícita 
en mis propias carnes. Desde entonces intento tomar en consideración aquello de lo que tuve experiencia en aquella época, tanto la experiencia positiva, como la negativa. Sobre todo me he puesto como tarea tomar de la manera más seria posible cada pregunta que se plantea en el aula, esto es, repetirla una vez más dándole la forma de interpretación más benévola para dar a entender al estudiante respectivo que su palabra cuenta y que tiene el derecho a una respuesta. Además presto mucha atención para conocer el trasfondo cultural de cada estudiante. En las tutorías siempre pregunto, a fin de indicar de este modo que soy consciente de las dificultades de la adaptación a un sistema cultural totalmente diferente. No hay más que decir, salvo dar a entender desde el principio a cada participante de una clase universitaria que su palabra tiene peso porque podría abrir perspectivas interesantes y alternativas de una interpretación creativa; es decir, hay que conceder algo así como un reconocimiento "anticipatorio" que indique la suposición de que se trata de un estudiante con mucho talento. Naturalmente, en este asunto también resulta importante no hacer ninguna diferencia entre los géneros o por la pertenencia cultural, e incluso contrarrestar, intentando sacar lo mejor de las manifestaciones de los representantes de una minoría.

F. H., B. H. \& M. R.: La idea del reconocimiento "anticipatorio" remite a la aplicación de la teoría del reconocimiento en la didáctica. En el caso de su aplicación a las distintas experiencias formativas nos parecería como si hubiera instituciones en las que este modo de reconocimiento estaría más fuertemente institucionalizado, como en el caso de las instituciones de los Estados Unidos. ¿Podría decirse que hay contextos en los que el reconocimiento mismo está más fuertemente reconocido? ¿sería esto algo así como un reconocimiento de segundo orden (Herzog, 2015) que precisarían las instituciones para poder ser reconocidas ellas mismas? ¿qué procesos sociales e históricos favorecerían, según su opinión, la constitución de instituciones formativas que producirían reconocimientos adecuados?

A. Honneth: El problema en la manera en la que ustedes formulan la pregunta es que en los contextos institucionales respectivos más bien resulta controvertida la cuestión de qué debe ser vigente como una forma "adecuada" del reconocimiento; solo las personas que hacen teoría social con un cierto coraje para tomar una posición previamente de carácter normativo o, en definitiva, el Estado de derecho legitimado democráticamente pueden formular, dentro de ciertos límites, qué modo de reconocimiento debería predominar en determinados sectores institucionales de una sociedad. Además, hay que distinguir mutuamente dos planos en el ejemplo de los centros educativos norteamericanos: por una parte, la imagen pública de los centros educativos, que más bien se consideran como instituciones que activan y son estimulantes para el rendimiento, y, por otra parte, los principios de reconocimiento establecidos en estas instituciones, que también allí se orientan más en la dirección de la posibilidad de una utilización económica que en 
la de la participación democrática, y ello a pesar de la influencia que John Dewey tuvo en el sistema educativo norteamericano. Respecto al primer plano, no estoy seguro de si se puede hablar de un "reconocimiento de segundo orden" o de si se trata más bien de una representación completamente básica de la función instructiva y del modo de actuación de los centros educativos, pero, aparte de esto, ustedes tienen ciertamente razón respecto de que la valorización o desvalorización de la importancia de una institución social, y por tanto de esta especie de "reconocimiento de segundo orden", puede contribuir enormemente al peso cultural de esa institución dentro de una sociedad, aunque ello no decide todavía, como quería indicar con la referencia al ejemplo norteamericano, sobre qué principios de reconocimiento predominan dentro de las instituciones respectivas. Les daré un ejemplo que quizá pueda aclarar esto. Durante un largo período de tiempo, en este plano del reconocimiento de segundo orden, la familia tuvo un peso enorme en Alemania, mucho más fuerte que en Francia, por ejemplo, pero las formas de reconocimiento que se mantendrían en estas familias y que serían valoradas públicamente serían ampliamente más reaccionarias que en muchos otros países. En síntesis, la valoración pública de una institución no nos dice nada sobre su cultura de reconocimiento interna.

***

F. H., B. H. \& M. R.: Al comienzo de su obra se encuentran dos experiencias, a nuestro entender relevantes para el campo de la educación: por un lado, su dedicación a los movimientos sociales y a los jóvenes (Clarke et al. 1979); por el otro lado, el análisis de la obra de Foucault (Honneth, 2009). Ahora bien, desde los principios de los años 80 se han producido muchos cambios. Por de pronto, la Unión Europea se plantea como objetivo para el año 2020, entre otros, que $90 \%$ de los jóvenes entre 18 y 24 años reciba una educación más allá de la educación secundaria. ¿̇Puede la ampliación de la etapa formativa ser una ayuda para los jóvenes a fin de desarrollar formas de expresión que les ayudan a luchar contra experiencias de desprecio, es decir, a fomentar el reconocimiento de los jóvenes? ¿O se podría decir con Foucault (y también con Bourdieu) que así se amplía el "dispositivo de control" y se asegura la reproducción del desprecio social ya existente?

A. Honneth: Déjenme también aquí ampliar en primer lugar un poco más el alcance de mi respuesta. Al principio de mi dedicación expresa a cuestiones de la educación se encuentra una participación activa en proyectos de "trabajo estudiantil", tal como se denominaba a principios de los años 1970, es decir, en la época en la que comencé en la Universidad de Bochum a dedicarme, además de la filosofía, también a la sociología. En estos proyectos voluntarios se intentaba precisamente interesar a personas de capas sociales más pobres, de entre 8 y 14 años, para que, con el consentimiento de sus padres, participaran en una enseñanza organizada por nosotros, al margen de la enseñanza escolar oficial, para aumentar sus posibilidades de concluir los estudios. Naturalmente, desempeñaba un papel en todo ello el ímpetu revolucionario y social del movimiento post-68. También queríamos enseñar 
a estos jóvenes procedentes de capas marginalizadas, amenazadas de desamparo y pobreza, a desarrollar un potencial de resistencia contra las condiciones existentes; y al mismo tiempo nos esforzamos también sinceramente en aumentar las perspectivas de éxito de aquellos alumnos y alumnas en sus respectivas escuelas, pues hacíamos lo que poco después se llamó enseñanza “compensatoria”, es decir, intentos intencionados de nivelar las dificultades de aprendizaje condicionadas por la socialización. Cuando me trasladé a la Universidad Libre de Berlín tuve que dejar el trabajo práctico con los jóvenes en tales proyectos de izquierda; pero mantuve el interés en este tema y me dediqué en el marco de mi trabajo en el Instituto de Sociología, junto con algunos de mis compañeras y compañeros, a cuestiones relativas a la socialización de jóvenes de la clase trabajadora. En el modesto estudio empírico que realizamos en aquel entonces en algunos barrios de Berlín, descubrimos lo que al mismo tiempo también se podía leer en los estudios del Center for Contemporary Cultural Studies en Birmingham, que en aquel entonces era dirigido por Stuart Hall ${ }^{1}$, a saber, que los jóvenes de las capas sociales obreras más pobres se programan, por así decir, de forma inconsciente, mediante sus prácticas culturales, para ser personas que fracasan en la escuela y así, en el futuro, proseguir el destino de sus padres poco formados; sobre esto, ayudan poco las buenas intenciones del personal docente, como recientemente mostró una vez más el sociólogo francés Didier Eribon en su importante estudio, de corte autobiográfico, sobre la generación de sus padres en el entorno obrero de Reims (Eribon, 2016, especialmente. pp. 44 ss). La lección que aprendió de forma temprana de ello era que, por regla general, está decidido el itinerario educativo del joven de clase trabajadora - y quizá se debería decir hoy en día: del joven de capas sociales más pobres con escasa formación - ya mucho antes de su ingreso en el colegio, y ello, mediante las condiciones de socialización del niño en su entorno social de procedencia. Esto significaría de forma práctico-política, que es preciso comenzar con la infancia muy temprana en cada intento de cambiar una suerte predestinada; tal como indiqué en un reciente artículo (Honneth, 2015), tenemos que intentar cambiar algo en las prácticas y representaciones liberales sobre la infancia, retirando el niño mucho más temprano de la soberanía educativa exclusiva de sus padres y "socializándolo" en instituciones preescolares con niños de la misma edad de otras capas sociales. De forma escueta se puede decir que las experiencias de desprecio que estos niños procedentes de capas sociales más pobres y con poca formación encuentran desde los primeros años de su vida (por el hecho de que perciben la arrogancia de unos compañeros suyos, la mirada denigrante de otros, etc., que no solo se dirige a su ropa o a su conducta tosca sino también a la falta de acceso al conocimiento "burgués" sobre el mundo), que estas experiencias de desprecio, digo, solo se podrían combatir si se cambiaran a fondo las condiciones sociales de la infancia; se podría decir incluso que los niños tendrían que adquirir experiencias ya desde muy temprano en espacios públicos adecuados a su edad en los que tales “distinciones" (véase también: Bourdieu, 1998) entre las clases no tuvieran un peso notable entre los participantes. La escuela misma 
siempre llega tarde, incluso cuando funciona de forma ideal como institución democrática según las recomendaciones de John Dewey. Con ello no quiero decir que resultarían superfluos los esfuerzos políticos por hacer un sistema escolar más justo del que tenemos, por ejemplo en Alemania. En cierto modo, hay que intentar cambiar la situación actual desde los dos lados, y por tanto romper el círculo vicioso de hogares con padres alejados de la formación y la pérdida posterior de oportunidades en el trabajo y en la profesión. Lo que resulta necesario es una "democratización" decisiva de la infancia y de la escuela.

$$
* * *
$$

F. H., B. H. \& M. R.: Sus explicaciones sobre la formación y la primera infancia nos parecen clarificadoras. Por otra parte, el análisis del capitalismo al modo de Thomas Piketty (2014) parecería favorecer con razón opiniones pesimistas. Afirma Piketty que menguará el papel de la formación en la movilidad social y se acrecentará el de la herencia económica. Una educación pública, animada por el reconocimiento anticipatorio, despertaría un potencial en las personas jóvenes y parecería desarrollarse en una dirección contrapuesta a las tendencias del capitalismo. ¿̇e podría decir que la formación, en su núcleo, en el sentido de la "democratización" que usted plantea, sigue una lógica anticapitalista?

A. Honneth: No creo ni en una "lógica" capitalista, ni en una relación fija de ajuste entre la economía capitalista e instituciones determinadas. Todo esto está mucho más disputado política y culturalmente de lo que aceptaría un análisis del capitalismo que procede hoy en la estela de los seguidores de Marx. Sin embargo, de este duro juicio, excluyo expresamente a Piketty, que mantiene que son posibles ciertas transformaciones institucionales dentro del espacio de nuestras sociedades capitalistas y, por ello, las recomienda con gran firmeza. Pero permítanme poner en claro lo que significa mi crítica aduciendo un ejemplo ilustrativo. Cuando estudiaba, en cualquier manual inspirado en el marxismo se leía sobre la socialización de la primera infancia que el capitalismo y la familia burguesa formaban una unidad irrompible; pero hoy en día, nos frotaríamos los ojos si en una lectura encontráramos tales afirmaciones y vemos actualmente que pueden existir familias patchwork o familias con padres homosexuales sin mayores dificultades en nuestra sociedad, que continúa siendo capitalista. Porque sería engañoso hablar de una "lógica" del capitalismo que solo permitiera instituciones de una determinada hechura. En el terreno de una economía capitalista son posibles muchas más alternativas institucionales en muchos sectores sociales de las que se pueden ver cuando se habla de una "lógica" del capital.

F. H., B. H. \& M. R.: La Teoría de la Acción Comunicativa (Habermas, 1995) se aplica a menudo en teorías y prácticas de educación. Habermas todavía tiene una gran influencia en las teorías de la educación en Alemania e Latinoamérica. A nuestro entender, esto se debe, por un lado al despliegue de una teoría de desarrollo moral que sigue a Kohlberg

400 Educação \& Realidade, Porto Alegre, v. 42, n. 1, p. 395-406, ene./mar. 2017. 
(en Habermas 1995, vol. 1: 250, vol. 2 260), y, por otro lado, al enfoque comunicativo que a menudo se relaciona con la pedagogía dialógica de Paolo Freire. ¿Por qué su crítica convincente a la Teoría de la Acción Comunicativa, ampliamente aceptada en otros ámbitos, parece tener poca influencia en las teorías de educación inspiradas en Habermas? ¿Qué significaría tomar en serio también para la teoría de educación su crítica a la comunicación verbal en Habermas?

A. Honneth: Permítanme primero corregir un poco su impresión. En los últimos años ha habido algunos cambios decisivos al respecto, al menos en la pedagogía alemana. Es cierto que la teoría habermasiana de la comunicación tenía al principio, es decir desde los años 80 , una especie de monopolio dentro de las ciencias de educación, pero la influencia disminuyó notablemente cuando comenzaron a surgir dudas respecto a la teoría de las etapas de desarrollo moral que seguía a Piaget. En el fondo, de nuevo creció lentamente la comprensión sociológica de que tales modelos de etapas dicen poco sobre la motivación efectiva de la acción moral y además prácticamente no hacen justicia a las diferentes formas sociales de lo moral. A la sombra de tales dudas crecientes se efectuó entonces dentro de la pedagogía -y aquí sólo puedo hablar de la situación alemana- una cierta pluralización. Por ejemplo la teoría de educación de Luhmann ganó influencia de forma considerable y lamentablemente también la investigación del cerebro, que en muchas disciplinas comenzó una marcha triunfal de corta duración. De todas formas, como consecuencia de esta pluralización también la Teoría del Reconocimiento ganó importancia de forma notable en las ciencias de educación, sobre todo, si lo contemplo de manera adecuada, en la pedagogía general, pero también en la investigación empírica sobre la escuela. En la medida en la que soy capaz de abarcar este interesante campo, se realizan desde hace algunos años investigaciones fascinantes sobre la negociación de reconocimiento entre profesores y alumnos por un lado y entre diferentes grupos de alumnos por el otro; en ello se sigue naturalmente la idea de que tales conflictos de reconocimiento se efectúan al principio por debajo del umbral de la articulación verbal y que giran alrededor de definiciones y atribuciones de "rendimiento", "competencia” y "éxito". Así se hace visible de repente la enseñanza escolar e incluso toda escuela como institución un trasfondo hasta ahora desatendido en buena medida, a saber la pugna de las personas implicadas -profesorado, alumnado y, lamentablemente también, padres y madres- por disponer de la autoridad para establecer criterios de rendimiento, objetivos de la enseñanza e incluso las tareas más generales de la escuela como tal. Todo ello está cambiando constantemente, pero solo se puede apreciar cuando se deja el nivel de la comunicación oficial y se enfoca al intercambio de gestos corporales, de señales silenciosas y del currículo "oculto", como se denomina en las investigaciones. Con ello, espero haber respondido a grandes rasgos a su pregunta.

***

F. H., B. H. \& M. R.: A lo largo de su obra detectamos que la cuestión de la educación y la formación no tiene un peso notorio. En su libro "El de- 
La Educación y la Teoría del Reconocimiento

recho de la libertad" (Honneth, 2014) analiza más profundamente la relación de las esferas de reconocimiento y las instituciones sociales. Por otra parte, en su "Réplica a la réplica" a Nancy Frazer afirma que "el sistema escolar estatal se integraría normativamente mediante dos ideas de reconocimiento social que compiten la una con la otra" (Honneth, 2003, p. 293). ¿Puede concretar un poco más la afirmación que hizo en este diálogo con Fraser? ¿Qué relación hay entre las diferentes esferas en el sistema escolar? ¿Cómo cambia esta relación en las diferentes etapas del sistema educativo?

A. Honneth: Tienen toda la razón del mundo. Hasta hace bien poco, las cuestiones relativas a la educación y la formación no habían tenido un papel destacado en mi teoría, aunque ello podría haberse esperado atendiendo a mis experiencias y dedicaciones descritas anteriormente. No sé yo mismo cómo debo explicar este vacío, que resulta claro si lo contemplamos en retrospectiva. Ciertamente, una razón es que en los años posteriores a 1968, cuando la educación jugó un papel muy importante desde puntos de vistas de lo más diverso - desarrollo psicosexual, procesos de socialización según la clase social, educación política, por mencionar solo algunos puntos -, tales preguntas desaparecieron de manera relativamente rápida del horizonte de atención de la filosofía, de la sociología y de la teoría política, y esta desaparición se produjo seguramente de forma errónea. Otra razón tiene que ver con el desarrollo de mis propias orientaciones teóricas que, como saben, se alineaban de manera cada vez más decidida con las ideas centrales de la filosofía social de Hegel. Aunque Hegel concedió una gran importancia a la noción de "formación" para su teoría del espíritu, no se dedicó más en detalle y de una forma adecuada a la realidad institucional de la educación pública ${ }^{2}$ - muy al contrario de Kant, que en este aspecto era mucho más "moderno"3. Si se echa un vistazo a la Filosofía del derecho de Hegel, entonces uno se da cuenta en seguida que en ella no se presta ninguna atención a la institución de la escuela, aunque toda su forma de proceder lo hubiera exigido; al fin y al cabo, Hegel quería reconstruir normativamente todas las instituciones sociales o representarlas según su idea de que deberían contribuir a la formación de la libertad individual dentro de la sociedad moderna; y a estas instituciones, en aquel entonces, ya pertenecería seguramente la escuela. De todas formas, creo que haberme dejado influir sin ninguna reflexión ulterior por esta negligencia del Hegel maduro de no conceder un peso importante a la educación y la formación organizadas públicamente, es un claro error en mi trabajo "El derecho de la libertad" cuyos resultados, por lo demás, defiendo todavía en su mayor parte. Así me explico mi propia omisión de no haber dado a la educación institucionalizada el importante papel que hubiera merecido seguramente en el marco de una teoría de la eticidad democrática.

Pero volviendo ahora al verdadero núcleo de la pregunta en torno a los dos principios de reconocimiento que están en competencia en el sistema público de educación. La idea que en nuestras sociedades las escuelas están determinadas por dos normas que compiten mutuamente, la adopté del sociólogo francés François Dubet (2000); este ha querido

402 Educação \& Realidade, Porto Alegre, v. 42, n. 1, p. 395-406, ene./mar. 2017. 
mostrar en una investigación muy bella que en las escuelas predomina tanto la norma de la "igualdad" como la norma del "rendimiento", sin que resulte establecido de una vez por todas de forma institucional cómo se deben relacionar la una con la otra. Esto me parece una idea altamente fructífera, que naturalmente se podría traducir con facilidad a mi teoría de reconocimiento: en nuestro sistema escolar se dirige a las alumnas y los alumnos o bien como a futuros ciudadanos, con la pretensión de que tengan las mismas posibilidades de participación y colaboración, o bien como a portadores de rendimientos en el mercado de trabajo, con todas las diferencias ya existentes en el correspondiente perfil del rendimiento. La misma distinción entre las dos formas de reconocimiento se puede describir también de manera que en el primer caso se comprende la enseñanza escolar como preparación a la formación de la voluntad democrática, mientras que en el segundo caso la enseñanza es entendida como cualificación pedagógica para el mercado laboral capitalista; en un caso, la escuela forma la bisagra institucional entre la socialización familiar y el Estado democrático de derecho, en el otro caso es la bisagra institucional entre la familia y el sistema económico. Sobre cuál de los dos principios de reconocimiento domina en la escuela en cada momento, deciden siempre, tal como lo veo yo, las disputas político-morales dentro del ámbito público acerca del papel y de la tarea de la educación escolar; y, según mi impresión, no hay duda de que hoy en día, en la ejecución de la llamada trasformación "neoliberal" del capitalismo occidental, el segundo principio de reconocimiento ha alcanzado el predominio, fuertemente apoyado por parte de los padres de las capas sociales más altas que así esperan ventajas competitivas para sus hijas e hijos en el mercado laboral. Por regla general quizá se podría decir que en épocas de reformas democráticas y de un mercado contraído siempre alcanza el predominio en el sistema escolar el primer principio de reconocimiento -como en los años 60 y 70-, mientras que en épocas del retroceso se impone el principio de rendimiento. Pero desde luego hay que tener cuidado con tales generalizaciones históricas.

F. H., B. H. \& M. R.: En su conferencia sobre "Educación y espació público democrático", usted reclama el desarrollo de formas de comunicación en la enseñanza "en las que las diferencias culturales no solo sean aceptadas de una manera lúdica, sino que puedan ser comprendidas como un enriquecimiento mutuo" (Honneth, 2012, p. 12). En muchos Estados occidentales la ampliación del sistema privado de educación, aparte de intereses económicos obvios, se justifica precisamente por el deseo de una pluralidad en las formas de educación. ¿Describiría esta forma de pluralidad como una patología social del sistema educativo que socava el potencial integrador de las instituciones públicas de educación?

A. Honneth: Mi respuesta a ello es un "sí" rotundo. Por varias razones soy muy escéptico respecto de la afirmación de que la ampliación de un sistema de escuelas privadas sirva para la pluralización de estilos educativos y, con ello, para la multiplicación de valores culturales. Pri- 
La Educación y la Teoría del Reconocimiento

mero hay que tener presente que las ideas de los valores y los objetivos educativos que se cultivan especialmente en tales escuelas privadas la mayoría de las veces son la expresión de ideas que tienen los padres sobre el futuro ético de sus hijos; en este punto, en la elección de la escuela privada, solo raras veces resulta efectivo el deseo autónomo de un niño o un joven; más bien son los padres quienes deciden qué tipo de educación axiológica deben recibir sus hijos en la enseñanza escolar. En este sentido la escuela privada aumenta las posibilidades de los padres de decidir sobre las preferencias futuras y las orientaciones axiológicas de sus hijos; este poder de socialización de los padres ya es lo suficientemente alto y no debería ser aumentado mediante la autorización estatal de escuelas privadas. Segundo, e igualmente evidente, es que un sistema de escuelas privadas está acompañado por el peligro de que la injusticia educativa existente en nuestras sociedades siga aumentando; y es que la asistencia a tales escuelas que no reciben financiación pública, por regla general, es muy cara así que solo los hijos de padres pudientes pueden "disfrutar" de ellas. Además hay que tener en cuenta que aquellos padres que han colocado a sus hijos en el "puerto" seguro de una escuela privada ya no van a abogar abiertamente por los asuntos del sistema escolar público; estas escuelas pierden, por lo tanto, un grupo importante de promotores potentes y poderosos. Se podría decir quizá que cuantas más escuelas privadas, tanto menor es el compromiso del espacio público democrático de disponer de un sistema sólido, lo más justo posible de educación pública. Y finalmente, tercero, el Estado de derecho abandona casi el único medio que tiene para crear en la futura generación las actitudes y orientaciones valorativas necesarias para la propia reproducción; solo las escuelas públicas se pueden ocupar de una forma generalmente controlada (mediante regulaciones para la participación y mediante la elección de métodos de enseñanza) para que sus alumnos adquieran los conocimientos y las orientaciones indispensables para una participación activa en la formación democrática de la voluntad. Como se ve, respecto a la propuesta que se extiende hoy en día de ampliar aun más el sistema de escuelas privadas, tengo las mayores objeciones, que proceden de mis convicciones democráticas.

F. H., B. H. \& M. R.: Para concluir queríamos volver una vez más a las condiciones de reconocimiento de las universidades. Tenemos la impresión de una cierta oposición precisamente en las universidades europeas frente a la influencia creciente de los rankings y las evaluaciones cuantitativas del trabajo de las personas docentes e investigadoras. ¿Podríamos entender el incremento de mediciones cuantitativas del rendimiento como una forma de búsqueda del reconocimiento o más bien como un ejemplo de una ideología o una patología del reconocimiento?

A. Honneth: En ello observo más bien la imposición agudizada de un determinado principio de reconocimiento en las instituciones formativas, a saber, la del rendimiento económicamente utilizable en detrimento del principio de la participación democrática y el compromiso participativo. Como parto de que la tarea de las instituciones formativas 
públicas es la de facilitar las sociedades constituidas democráticamente, y especialmente las capacidades de la cooperación y el intercambio intelectual, contemplaría los desarrollos mencionados como una patología social, que sin duda ha sido puesta en curso por la ideología del neoliberalismo.

F. H., B. H. \& M. R.: Muchas gracias por esta conversación, profesor Honneth.

Recibido en 20 de octubre de 2016 Aprobado en 22 de diciembre de 2016

\section{Notas}

1 Stuart M. Hall fue director del Centre for Contemporary Studies de la Universidad de Birmingham entre 1972 y 1979.

2 Aquí, siguiendo el uso del término en Kant o Hegel, educación «pública» se contrapone a educación familiar o doméstica.

3 Kant dedicó a la educación un curso en la Universidad de Königsberg, publicado con el título "Pedagogía", en el que introduce las ideas de Rousseau en su filosofía trascendental. Acuñó la expresión "teoría de la educación”.

\section{Referencias}

BOURDIEU, Pierre. La Distinción. Criterio y bases sociales del gusto. Trad. de Ma. del Carmen Ruiz de Elvira. Madrid: Taurus, 1998.

CLARKE, J. et al. Jugendkultur als Widerstand: Milieus, Rituale, Provokationen [Cultura juvenil como resistencia: Medios, rituales, provocaciones]. Edic. Axel Honneth. Trad. al alemán Thomas y Susi Buttel. Frankfurt d. M.: Syndicat, 1990. DUBET, François. L'égalité et le mérite dans l'école démocratique de masse. L'Année sociologique, v. 50, n. 2, p. 383-408, 2000.

ERIBON, Didier. Rückkehr nach Reims. Berlín: Suhrkamp, 2016.

HABERMAS, Jürgen. Theorie des Kommunikativen Handelns. Fráncfort d. M.: Suhrkamp, 1995.

HERZOG, Benno. Recognition in multicultural societies: intergroup relations as second-order recognition. Revista Internacional de Sociología, Córdoba, v. 73, n. 2, p. 1-12, 2015.

HONNETH, Axel. Crítica del Poder: fases en la reflexión de una teoría crítica de la sociedad. Madrid: A. Machado Libros, 2009.

HONNETH, Axel. Erziehung und demokratische Öffentlichkeit. Ein vernachlässigtes Kapitel der politischen Philosophie. Zeitschrift für Erziehungswissenschaft, v. 15, n. 3, p. 429-442, 2012.

HONNETH, Axel. El Derecho de la Libertad. Esbozo de una eticidad democrática. Madrid: Katz; Clave Intelectual, 2014.

HONNETH, Axel. Kindheit. Unstimmigkeiten unserer liberalen Vorstellungswelt. In: BETZLER, Monika; BLEISCH, Barbara (Ed.). Familiäre Pflichten. Berlín: Suhrkamp, 2015. P. 147-174.

Educação \& Realidade, Porto Alegre, v. 42, n. 1, p. 395-406, ene./mar. 2017. 
HONNETH, Axel; FRASER, Nancy. Umverteilung oder Anerkennung? Eine politischphilosophische Kontroverse. [¿Redistribución o reconocimiento? Una controversia de filosofía política]. Fráncfort d. M.: Suhrkamp, 2003.

PIKETTY, Thomas. El capital en el siglo XXI. México: Fondo de Cultura Económica, 2014

Francesc J. Hernàndez I Dobon es profesor del Departamento de Sociología y Antropología Social de la Universidad de Valencia.

Email: francesc.j.hernandez@uv.es

Benno Herzog es profesor del Departamento de Sociología y Antropología Social de la Universidad de Valencia.

Email: benno.herzog@uv.es

Maurício Rebelo Martins es Doctorando en Educación en la Universidade Estadual de Campinas y Bolsita FAPESP.

Email:maurebelo@gmail.com 